\title{
Differential stimulated infrared radiometry: application to remote detection of cracks
}

\author{
by A.C. BOCCARA $(*)$, D.FOURNIER $(* *)$, \\ J. GUITONNY $(* *)$, M. LE LIBOUX ${ }^{(*)}$ and A. M. MANSANARES $(* *)$
}

(*) Laboratoire d'Optique ESPCI - UPRA O0O5 du CNRS, ESPCI, 10 rue Vauquelin, F-75005 Paris.

(**) Laboratoire d'Instrumentation UPMC - UPRA 0005 du CNRS, ESPCI, 10 rue Vauquelin, F-75005 Paris.

\begin{abstract}
We present a new approach for remote detection of cracks using a modulated local excitation and an infrared detection. In order to improve the contrast of the detection signals and to reveal more easily the dissymetry of the temperature field in the heated area close to a crack, we have used a differential detection. This set up whose rejection of the average signal is of the order of $10^{2}-10^{3}$ allows an almost zero background detection.
\end{abstract}

\section{Introduction}

It is a classical task for Non Destructive Evaluation scientists and engineers to detect cracks and delaminations. Photothermal techniques are now well established for this purpose because such defects disturb the heat flow. When the defects are parallel to the sample surface, the surface temperature is modified and a careful analysis of the signals in the time (or frequency) domain reveal the depth and the size of the defect.

When the excitation is uniforme (for instance, flash excitation) it is difficult or impossible to detect slanted or vertical cracks. By using a local excitation, a deformation of the temperature field appears near this kind of defects. Using the mirage effect and the transverse detection of the deflexion, the Wayne State University Group [1] has demonstrated this symmetry breaking. We have used the same technique to check the quality of inner laser welding [2].

For a remote detection it has been proved that IR detection is easier to implement [3]. For this reason we have built a set up using infrared radiometry but in a real time differential scheme.

In a first part we will describe the experimental set up and its limits. The physical basis appear in a second part and some preliminary results in a third one.

\section{Experimental set up}

Figure 1 describes the experimental scheme. The sample is illuminated by a modulated Argon laser whose average power is in the $100 \mathrm{~mW}-1 \mathrm{~W}$ range. This laser is carefully focussed on the sample, the spot diameter being a few tens of micrometers. The samples under investigation being opaque for this wavelength the energy is thus deposited on the surface. The IR emission is collected by a spherical mirror, and focussed on the detector. The sample and the detector are located near the center of the mirror in order to reduce the aberrations (applanetic system). The size of 
each $\mathrm{HgCdTe}$ (Judson) detector is $250 \times 250 \mu \mathrm{m}^{2}$, the gap between them is $30 \mu \mathrm{m}$. The maximum efficiency is in the $8-12 \mu \mathrm{m}$ range. The preamplifier (DC - $1 \mathrm{MHz}$ ) gains are separately adjustable in order to balance the overall response of the system. The signal processor is a lock in amplifier. Both the acquisition process and the sample displacement are driven by a computer.

On a non-prepared "Invar" sample the signal over noise ratio on one detector is $10^{3}$, while this ratio can reach $10^{5}$ for blackened surface. The rejection between the two channels is in the $10^{2}$ to $10^{3}$ range. Let us point out that a small drift of this balanced differential signal can appear correlated with the unfocussing of the spot when scanning the sample.

\section{Physical basis}

The modelisation needed to take advantage of the experimental setup possibilities concerns both the evaluation of the thermal parameters and the description of the effects of a thermal barrier on the temperature field.

It is well known that the surface temperature exhibits strong phase and amplitude variations when going from a 3-D regime (low frequency modulation range for which the thermal diffusion length is much larger than the heating spot size) to a 1-D regime (high frequency range for which the thermal diffusion length is much smaller than the heating spot size). This behaviour is illustrated on figure 2 for a $30 \mu \mathrm{m}$ radius excitation spot and a $150 \mu \mathrm{m}$ radius detector and two values of thermal diffusivity. A best fit procedure can be used to determine the thermal diffusivity of the sample under investigation.

In a first approach to modelize the effects, we have used the model developped by MCDONALD [ 4 ] which describes the temperature field when scanning accross a vertical crack. The figure 3 describes the local temperature on the center of the heated area. When scanning through the defect, the calculation for a $100 \mathrm{~Hz}$ modulation frequency and a thermal diffusivity of $0.025 \mathrm{~cm}^{2} / \mathrm{s}$ has been performed with different values of the product $R_{T} k_{S}\left(R_{T} k_{S}=0,10^{-3}, 10^{-2}\right.$ and $1 \mathrm{~cm}$, where $R T$ is the thermal resistance and $k_{S}$ is the sample thermal conductivity) for a $30 \mu \mathrm{m}$ radius Argon laser excitation. Let us notice that in our case we have to make an average of the heated area conjugated to each detector.

\section{Experimental results}

A first set of experiments has been carried out on a steel sample exhibiting an artificial defect: a $400 \mu \mathrm{m}$ slit was machined in the section, in order to simulate a closed subsurface crack, perpendicular to the sample surface. On the figure 4 we can compare a direct infrared detection signal with a differential one. The defect can be detected on the single detector experiment, but the signal itself is of the order of the drift during the scanning. The differential detection has enhanced the contrast and the position of the defect is clearly associated to the $S$ shaped signal. This signal can be considered as the signature of a vertical closed crack in a sample. 


\section{Conclusion}

The experiments carried out in the differential mode have cleary demonstrated their sensitivity to the symmetry breaking of the temperature field. In particular they have revealed the signature of a vertical crack with a much better contrast than the usual detection scheme.

We are now going to apply this technique to other crack geometries (slanted). We strongly believe that such approach can be coupled to an automatic defect signature processing system using neural networks that we have proposed recently.

Moreover this detection will also be coupled with the so called flying spot geometry in which the excitation is tracked by the detection signal [ 5] in order to get highly contrasted images.

\section{REFERENCES}

[1] GRICE (K.R.), INGLEHART (L.J.), FAVRO (L.D.), KUO (P.K.) and THOMAS (R.L.).Thermal wave imaging of closed cracks in opaque solids. J.Appl.Phys., 54 (11), 1983, p. 6245.

[ 2 ] LEPOUTRE (F.), FOURNIER (D.) and BOCCARA (A.C.).- Non destructive control of welding using the mirage detection. J.Appl.Phys., 57, 1985, p. 1009.

[ 3.] BUSSE (G.) and WALTHER (H.G.).- Photothermal non destructive evaluation of materials with thermal waves. Elsevier, Andreas Mandelis Ed., 1992, p. 205-298.

[ 4 ] MCDONALD (F.A.), WETSEL (G. C. Jr.) and JAMIESON (G. E.).- Photothermal beam-deflection imaging of vertical interfaces in solids. Can. J. Phys., 64, 1986, p. 1265.

[5] WANG (Y.Q.), KUO (P.K.), FAVRO (L.D.) and THOMAS (R.L.)-- A novel Flying-Spot Infrared camera for imaging very fast thermal-wave phenomena. Springer Series in Optical Sciences, 1990, p. 24-26.

\section{Acknowledgments}

We thank the MRT for financial support and CEDIP for its collaboration. A.M.M. thanks the CNPq (Brazil) for a post doctoral grant. 


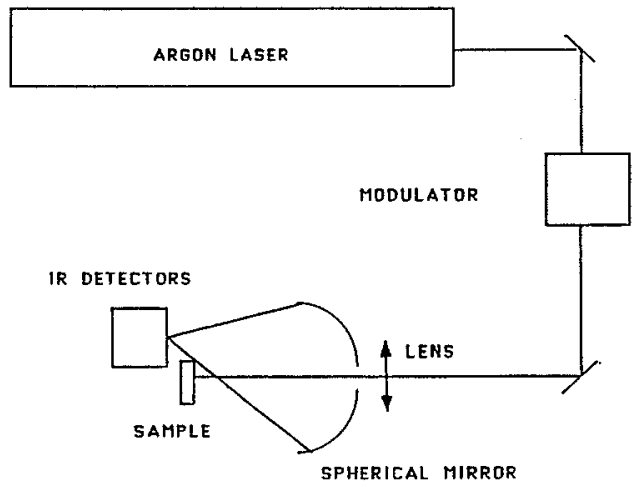

Fig.1. - Scheme of the experimental set up.
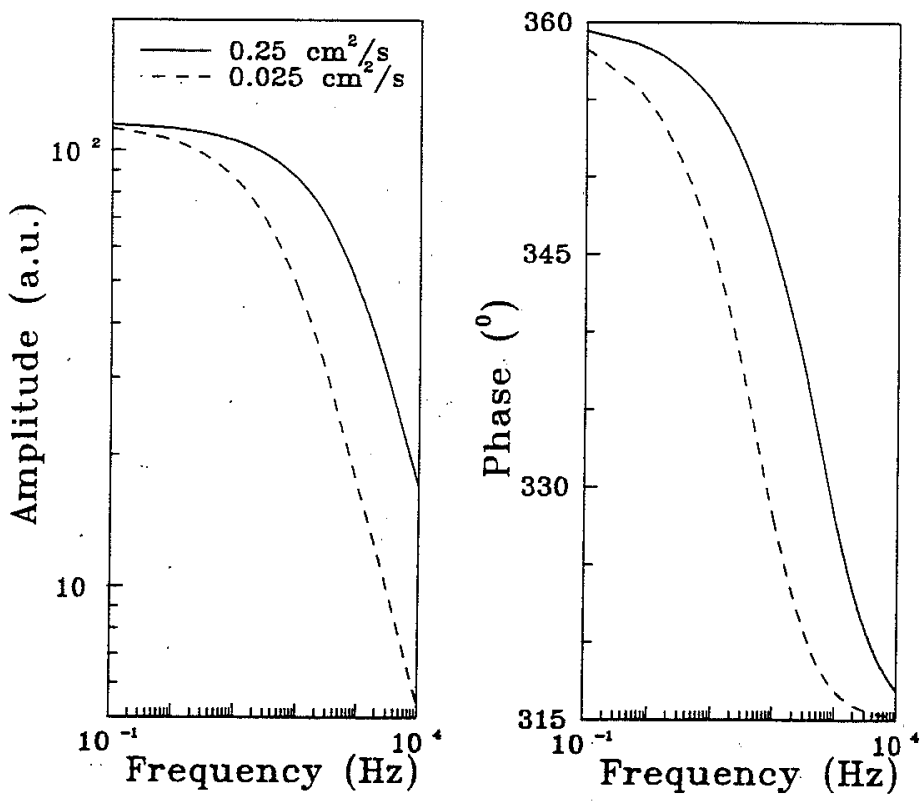

Fig.2. - Theoretical calculation of the amplitude and phase of the average temperature on the sample surface, for an homogeneous sample, as a function of the modulation frequency, for two values of the sample thermal diffusivity. 
http://dx.doi.org/10.21611/qirt.1992.060
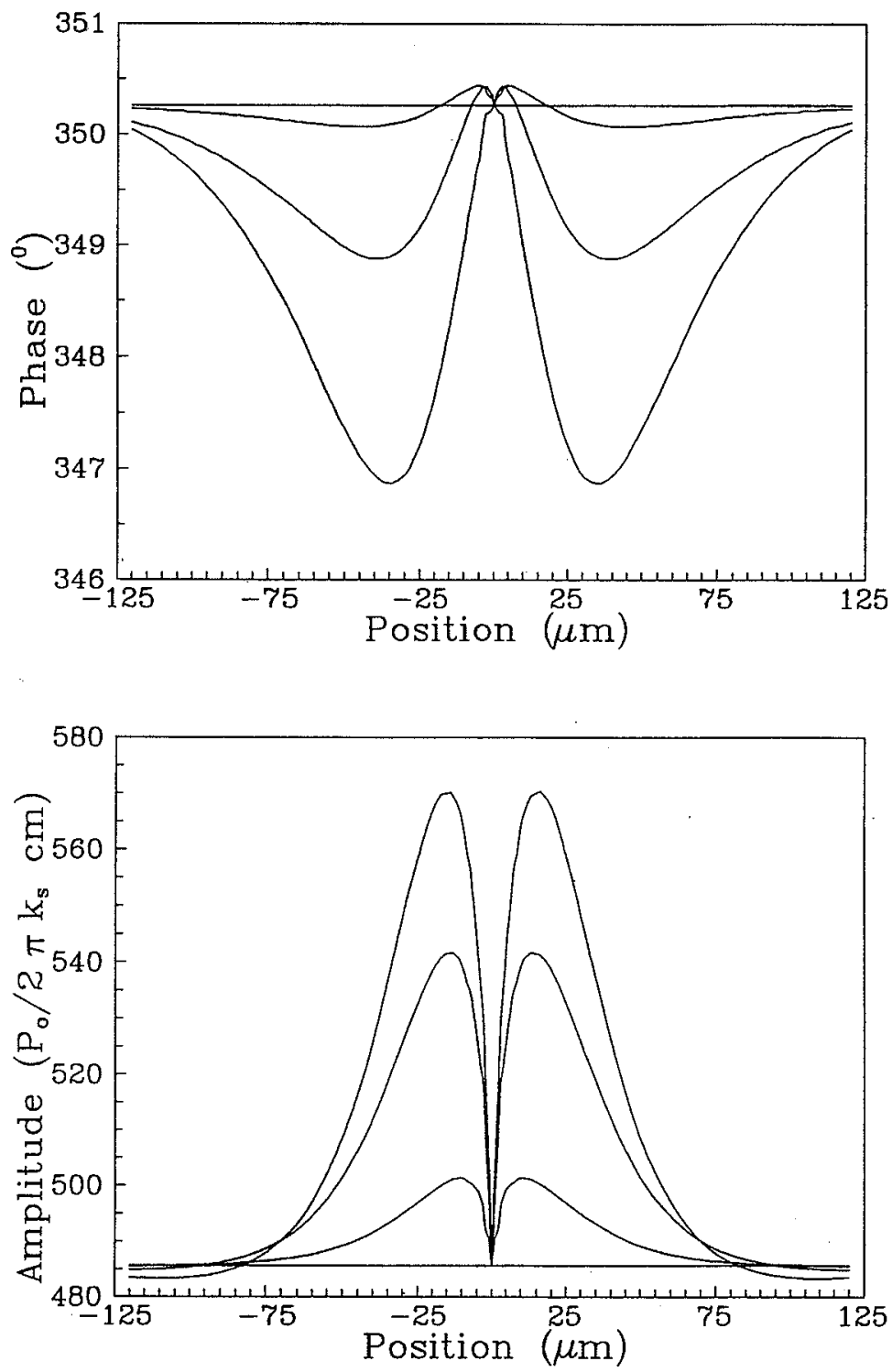

Fig.3. - Theoretical calculation of the amplitude and phase of the local temperature on the center of the pump beam, when scanning through the vertical defect at position equal to zero. The temperature perturbation increases with the value of $\mathbf{R}_{\mathbf{T}} \mathbf{k}_{\mathrm{S}}$. 

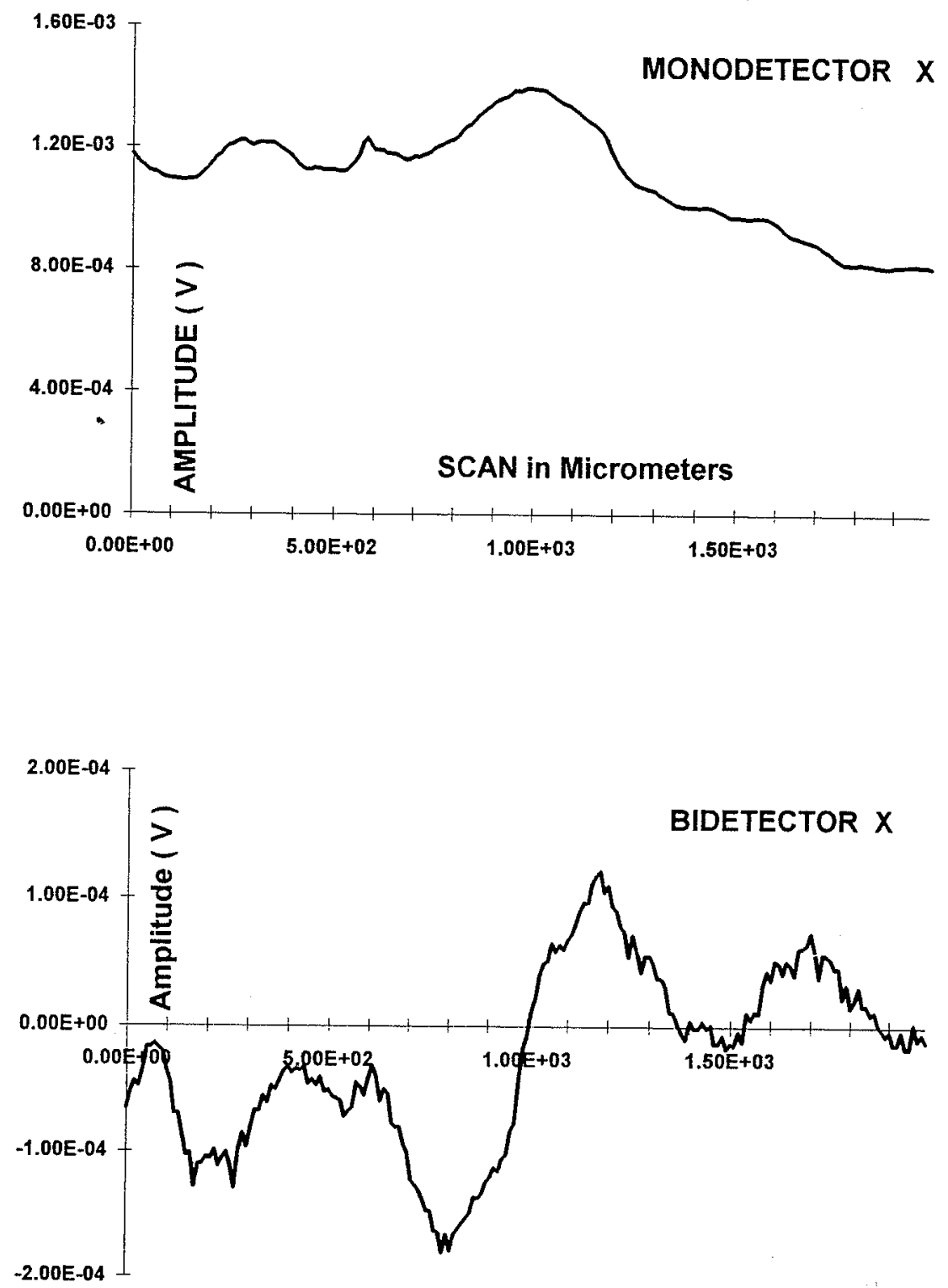

SCAN in Micrometers

Fig.4 - Experimental curves for a direct (monodetector) and a differential (bidetector) infrared detection, when scanning a steel sample exhibiting an artificial vertical subsurface defect. 